\title{
Peer Review and Darwinian Selection
}

\author{
Kostas Kampourakis $^{1}$ - Ross $\mathrm{Nehm}^{2}$ - Alice S. L. Wong ${ }^{3}$. \\ Charbel N. El-Hani ${ }^{4}$
}

Published online: 3 November 2015

(C) Springer Science+Business Media Dordrecht 2015

Charles Darwin is known for developing one of the first well-documented evolutionary theories in his book On the Origin of Species, published in 1859. Although Darwin's "long argument" included several conceptual advances, most notably natural selection and the tree of life, his theory came to be widely known as "the theory of evolution by natural selection." Darwin continually revised On the Origin of Species in consecutive editions, and as he did so he eventually adopted a conceptualization of selection that was significantly different from his initial one. Darwin initially thought of natural selection as a creative process that produced organisms adapted to their local environment through the accumulation of slight variations over many generations. However, he later adopted the conceptualization of the "survival of the fittest," according to which natural selection was an eliminative process that only allowed the most well-suited individuals (the "fittest") to survive and reproduce. Therefore, whereas Darwin began with a conceptualization of a process of selection for traits, he continued with a conceptualization of selection against traits.

If we manage to overlook this oversimplified and anthropomorphized synopsis of a rich body of work, these two distinct conceptualizations of selection have important differences between them. The first conceptualization describes a gradual selection process through which several variable members of a population coexist; those that eventually survive and reproduce are those that for whatever reason come to have a beneficial combination of characters through selection over consecutive generations. This is selection for traits, as the emphasis here is on those beneficial traits that eventually become prevalent in the population. In contrast, the second conceptualization describes a relatively fast selection process through which all but a few exceptional members of a population, already possessing a

Kostas Kampourakis

Kostas.Kampourakis@unige.ch

1 University of Geneva, Pavillon Mail, 40 Boulevard du Pont-d'Arve, 1211 Geneva, Switzerland

2 Stony Brook University (SUNY), Stony Brook, NY, USA

3 The University of Hong Kong, Hong Kong, China

4 Federal University of Bahia, Salvador, Brazil 
beneficial combination of characters, are eliminated. This is selection against particular (less-"fit") traits that are eliminated through the death of their bearers. A simple analogy can help us visualize these different processes: In the second case, there is a filter through which only some already established objects can pass; in the first case the objects are molded along the way, and some thus happen to be able to pass through the filter. Therefore, the important difference here is in what condition the objects reach the filter. In the second case, the objects are already molded, and they either make it or not in their instantaneous interaction with the filter; in the first case there is a more long-term interaction with the filter, and so if they are remolded again and again, and eventually change, objects can move through it.

The peer review process can be one of the most subjective endeavors in the scholarly world. It should not be, and it does not have to be subjective, but it can be. Each reviewer has his/her own conceptualizations, views, experiences, and biases, which can collectively impact the stance taken toward a manuscript. Therefore, it is the role of the editors to address these issues and eventually attempt to deliver a fair and constructive set of reviews to the authors. This is of course possible, but it also depends on what the editors anticipate from the reviewers. Is it only a recommendation for an editorial decision, grounded in rigorous analysis and thoughtful argument? Or is it more than that? In Science \& Education, we take the latter view. If we relied on reviewers only to make an editorial decision, then we would be implementing a process similar to the selection against one described above. The reason for this is that most manuscripts are not appropriate for publication when we initially receive them. They always have limitations, which authors themselves are unable to identify - we know this from our own publication experiences. Therefore, if the editors only relied on reviewers for a decision, this would most likely be a "reject" one in the first place.

However, we believe that reviewers have a very creative role to play and contribute to a process of selection for (manuscripts in this case). Reviewers are always experts in their domains, and when their review is constructive, it provides crucial feedback to authors. This is why reviewers of Science \& Education (and of other journals of course) have always submitted detailed reviews with specific suggestions that most often aim to help authors effectively revise their work in order to make it publishable. This results in a "major revisions" decision that does not guarantee acceptance, yet indicates that insofar as the suggested revisions are made, the revised manuscript will be considered for publication. In order to enhance the creative and constructive role of the reviewers, we ask authors to submit the revised manuscript using a color code, by which they indicate which revisions were made in response to which comments. Thus, by using a single color for each reviewer, it becomes immediately clear which parts of the manuscript have been revised and which suggestions they have incorporated. Authors also submit a detailed list of responses to the reviewers' comments. As a result, the manuscript is "molded" based on how the authors use the reviewers' suggestions. This is the creative aspect of the selection process.

When a revised manuscript and the authors' responses to the reviewers' comments are submitted to the journal, we immediately invite the initial reviewers to re-review them. We are aware that this is time consuming, both for the review process and for the reviewers themselves. Yet, we believe that the expert reviewers can better decide not only whether the requested revisions were appropriately made, but also what else needs to be done or might be done in order to ensure a high-quality manuscript. In situations where the reviewers decline our invitation, which thankfully are uncommon, we invite new reviewers who are experts in the same domain. They are asked to review the revised manuscript and 
also comment on how effectively the initial reviewers' suggestions were taken into account and implemented in the revised manuscript. Only after the reviewers of the revised version recommend acceptance do we begin to consider the manuscript for publication. Then, our own editing begins, making suggestions for further improvement to the manuscript and for clarifying particular points. Finally, after a lot of work on behalf of the authors, the reviewers and the editors a manuscript is accepted for publication.

We must note that in our journal reviewers are drawn from different fields and, more often than not, the reviewers of the same manuscript bring different kinds of expertise. The reason for this is that the manuscripts submitted to Science \& Education should draw on literature from history, philosophy and sociology of science, as well as on the relevant science education literature. The manuscripts should also have explicit and clear implications for science teaching and learning. Therefore, as we invite three reviewers for each manuscript, we may attempt to enlist a historian, a philosopher, and an education researcher to comment on a given manuscript. Of course, people cannot be easily categorized in most cases, as many of our reviewers work in more than one field (e.g., history of science and science education). However, what we want to highlight here is that the reviews received come from different perspectives. This can be very useful to authors, particularly as they are not experts in all fields.

We refrained from mentioning all the details of manuscript processing, such as the initial scanning of manuscripts, during which we reject without review the ones that clearly fall outside the scope of the journal. Our aim in this editorial is to emphasize that we are relying on reviewers in order to help authors improve their work as much as possible. We are thus adopting the stance of selection for (potential) high-quality manuscripts rather than selection against (currently) low-quality manuscripts. Therefore, if you are a prospective author, you should know that we will help you as much as we can to improve the quality of your work so that it has broad significance for the field. If you are a prospective reviewer, we will rely on you to be a constructive and helpful partner. Let us all work creatively together! 